\title{
PROPIEDADES PSICOMÉTRICAS DE LA ESCALA RIESGO SUICIDA DE PLUTCHIK EN UNA MUESTRA DE JÓVENES MEXICANOS PRIVADOS DE LA LIBERTAD
}

Psychometric properties of the Plutchik suicide risk scale in a sample of young Mexicans deprived of liberty

\author{
Marco Antonio Santana-Campas*, Felipe Santoyo Telles**
} Universidad de Guadalajara

\section{Resumen}

Objetivo: reportar propiedades psicométricas de la escala de riesgo de suicidio de Plutchik en muestra penitenciaria. Método: investigación cuantitativa no experimental y correlacional. Muestra: 395 jóvenes privados de la libertad de cinco instituciones penitenciarias del estado de Jalisco, México, $84.1 \%$ hombres y $15.9 \%$ mujeres con rango de edad de 15 a 29 años. Instrumento: escala de riesgo de suicidio de Plutchik. Resultados: alfa de Cronbach de la escala .749 , el análisis factorial confirmatorio mostró un ajuste optimo, la raíz cuadrada media de error de aproximación (RMSEA <.06) fue de .065, la razón de chi-cuadrado sobre los grados de libertad (CMIN/DF <3) fue de 2.66 y el índice de bondad de ajuste (GFI > .9) fue de .927. Esta escala demostró adecuadas propiedades psicométricas, por lo que resulta ser un instrumento idóneo para identificar el riesgo de suicidio.

Palabras clave: Riesgo de suicidio, jóvenes prisioneros, evaluación, suicidio.

\begin{abstract}
The objective was to report the psychometric properties of the Plutchik Suicide Risk Scale in prison sample. Method, non-experimental and correlational quantitative research. Sample: 395 young people deprived of freedom, $84.1 \%$ men and $15.9 \%$ women, the age range was 15 to 29 years. It was taken from five penitentiary institutions in Jalisco, Mexico. Instruments: Plutchik Suicide Risk Scale and Beck's Hopelessness Scale. Results, the Cronbach's alpha of the scale was .745, the confirmatory factorial showed an optimal fit, the approach's error average square root (RMSEA <.05) was .05, the chi-square ratio on the degrees of freedom $(C M I N / D F<3)$ was 1.99 and the goodness of fit index (GFI> .9) was .949. This scale demonstrated adequate psychometric properties, which is why it is an ideal instrument to identify the risk of suicide.
\end{abstract}

Keywords: Suicide risk, young prisoners, evaluation, suicide.

* Psicólogo. Fiscalía de Reinserción Social de Jalisco. Centro Universitario del Sur de la Universidad de Guadalajara, Jalisco, México. marco.scampas@academicos.udg.mx

** Ingeniero. Centro Universitario del Sur, Universidad de Guadalajara, Jalisco, México. 


\section{INTRODUCCIÓN}

El suicidio se ha convertido en un problema de salud pública, en el mundo, supuso el 1,8\% de la carga global de mortalidad en 1998, y en el 2020 representará el 2,4\%. Los grupos considerados vulnerables son los que representan mayor riesgo de suicidio, entre estos grupos, están las personas privadas de la libertad, hay más de 10 millones presos, estos están en mayor riesgo de mortalidad por suicidio, autolesiones y violencia (Fazel, Hayes, Bartellas, Clerici \& Trestman, 2016).

Fazel, Ramesh y Hawton (2017) reportaron que ocurrieron 3906 suicidios en prisiones en el período del 2011 al 2014 en las regiones de ingreso alto: Europa, Norteamérica y la región de Australasia, la tasa de suicidio en prisión es de más de 100 por 100000 presos por año (Fazel et al. 2017). En Francia Duthé, Hazard y Kensey (2014) mediante un estudio de prevalencias de suicidios en cárceles durante el periodo de 2006 al 2009, reportaron que en las cárceles se suicidan 17.2 hombres y 13.6 mujeres por cada 10,000 presos. En Alemania, Radeloff et al. (2015) realizaron un estudio en el que obtuvieron datos del periodo del 2000 al 2010 , reportaron que el riesgo relativo de suicidio en jóvenes privados de la libertad fue del 23.0 y en adultos prisioneros fue de 7.7. Además del suicidio, en la población penitencia, la prevalencia de trastornos mentales es mayor que en la población en general, siendo esto el principal factor de riesgo para el suicidio (Fazel et al. 2016).

La National Action Alliance for Suicide Prevention (2013) informó que en Estados Unidos de Norteamérica entre el 8 y el $21 \%$ de los jóvenes a disposición en los centros de justicia para menores, presentaron ideación suicida en los últimos seis meses y entre el 19 y el 29\% en el último año. Además, entre el 1.4 y el $3 \%$ había intentado suicidarse en el último mes y entre el 9.5 y el $15 \%$ había intentado suicidarse en el último año.

En las cárceles de México, durante el año 2017 se registraron 632 autoagresiones, 42 intentos de suicidio y 98 suicidios, al mes de abril del 2018, se han registrado 160 Autoagresiones, 20 intentos de suicido y 28 suicidios. Lo anterior de acuerdo con el observatorio de prisiones (Observatorio de Prisiones, 2018).
De acuerdo con Marzano et al. (2016) los factores asociados a los intentos de suicidio de los presos incluyen una serie de factores clínicos, psicosociales y ambientales potencialmente modificables. Por otra parte, Glenn y Nock (2013) argumentaron la falta de investigaciones que proporcionen los factores predictivos e inferenciales del riesgo de suicidio que sirvan como base para la prevención, e incluir en las investigaciones la amplia gama de las variables sociodemográficas, trastornos de personalidad, y las variables psicosociales.

Para el estudio de los factores que inciden el suicidio, y en miras de prevenirlo, se creó el concepto de riesgo de suicidio, este se entiende como todo acto encaminado a infringirse daño a uno mismo, de forma deliberada, independientemente del grado de letalidad, incluye las conductas autolesivas, la ideación y el intento previo de suicidio (Beck, Weissman, Lester \& Trexler, 1974).

Por otra parte, Plutchik y Van Praag (1989), en la creación de la escala de riesgo de suicidio, consideraron relevante incluir reactivos que ayuden a identificar: historia pasada de intentos de suicidio, impulsos y planes dirigidos hacia la autodestrucción, sentimientos de desesperanza, depresión, uso frecuente de tranquilizantes, pastillas para dormir y medicamentos de todo tipo. Concluyeron que la escala tenía fiabilidad interna (.84) y que las puntuaciones en la escala discriminaron entre un grupo de pacientes ambulatorios evaluados clínicamente como síntomas suicidas y un grupo de pacientes que no presentaron síntomas suicidas. También discriminaba entre los pacientes que habían hecho un intento de suicidio en el pasado y aquellos que nunca habían hecho tal intento.

En la identificación de los factores que influyen en el riesgo de suicidio, se ha encontrado como limitante una variedad de instrumentos para medir estos factores y para medir el riesgo de suicidio, creando con esto una variedad de términos e instrumentos (Marzano et al. 2016, National Action Alliance for Suicide Prevention, 2013).

Dentro de la literatura se encontró una variedad de instrumentos para identificar el riesgo de suicidio: Scale for Suicide Ideation (por sus siglas en inglés: 
SSI; Beck, Kovacs \& Weissman 1979), Self-Rated Scale For Suicide Ideation (por sus siglas en inglés: SR-SSI; Beck, Steer y Ranieri, 1988), Modified Scale For Suicide Ideation (por sus siglas en inglés: MSSI; Miller, Norman, Bishop \& Dow, 1986), Plutchik Suicide Risk Scale (Plutchik \& Van Praag, 1989), Suicide Assessment Scale (por sus siglas en inglés: SUAS; Stanley, Traskman-Bendz \& Stanley, 986), Modified Suicide Assessment Scale y Suicide Assessment Scale Self Rated (por sus siglas en inglés: SUAS-S; Niméus, Ståhlfors, Sunnqvist, Stanley \& Träskman-Bendz, 2006), Suicide Intent Scale (por sus siglas en inglés: SIS; Beck, Schuyler \& Herman, 1974), Adult Suicidal Ideation Questionnaire (por sus siglas en inglés: ASIQ; Osman et al. 2005) e Inventario de suicidio Orientación-30 (por sus siglas en inglés: ISO30; Rey \& Kowalchuk, 1994).

De los instrumentos que se describen con anterioridad, los más utilizados en contexto penitenciario son la escala de riesgo de suicido de Plutchik (Aristizábal et al. 2013; Calvo-García et al. 2016; Jiménez \& Linero, 2015; Marzano et al. 2011; Mojica et al. 2009), el Inventario de suicidio Orientación-30 (Cañón et al. 2015; Jaramillo et al. 2015), estos autores han realizado las adaptaciones necesarias para esta población, pero ninguna de estas investigaciones se ha realizado en México.

De acuerdo con Rangel-Garzón, Suárez-Beltrán y Escobar-Córdoba (2015), la Escala de Riesgo Suicida de Plutchik surgen como instrumento oportuno y confiable para la evaluación del riesgo suicida. Además, la escala de riesgo suicida de Plutchik es de fácil comprensión, está compuesta de 15 reactivos y sus opciones de respuesta son sí o no. Considerando las características socioculturales y académicas de los jóvenes prisioneros, la escala de riesgo suicida de Plutchik resulta idónea para esta población, por lo tanto, es la que se utilizó en esta investigación.

Asimismo, resulta relevante precisar, que, de los instrumentos mencionados con antelación, ninguno fue diseñado para población penitenciaria, misma que se encuentra en condiciones microsociales, legales e institucionales diferentes a las de la población general, donde los instrumentos tienen la finalidad de prever de herramientas a los clínicos, investigadores y profesionales de la salud pública, y no precisamente aplicable a población penitenciaria, este acotamiento también lo menciona la American Psychiatric Association en la Guía de Consulta de los Criterios Diagnósticos Versión 5 (por sus siglas en inglés DSM5; APA, 2014) sobre el uso y aplicación de los criterios diagnósticos en población forense.

Por lo anterior, el objetivo de esta investigación es reportar las propiedades psicométricas de la escala de riesgo de suicidio de Plutchik.

\section{MÉTODO}

Esta es una investigación cuantitativa no experimental y correlacional, puesto que no existe manipulación o control de las variables y se busca determinar las propiedades psicométricas de la escala de riesgo de suicidio de Plutchik y la correlación con la desesperanza.

\section{MUESTRA}

Está conformada por 395 jóvenes privados de la libertad, misma que se tomó de cinco de las 13 instituciones penitenciarias del Estado de Jalisco, México. El $84.1 \%$ fueron hombres y el $15.9 \%$ mujeres, el promedio de edad fue de 23.78 con un rango entre 15 a 29 años. El estado civil predominante fue soltero $51.9 \%$ seguido de concubinato $32.7 \%$. La escolaridad referida en mayor proporción fue de educación secundaria con el $47.1 \%$ seguido de educación primaria con el $26.3 \%$.

\section{INSTRUMENTO}

Escala de Riesgo Suicida de Plutchik (Plutchik, Van Praag, Conte \& Ricard, 1989). Validación española de Rubio et al. (1998) y la revisión de Sábado y Monforte (2010). Se trata de un cuestionario autoadministrado de 15 preguntas, con respuesta si/no. Cada respuesta afirmativa puntúa 1. El total es la suma de las puntuaciones de todos los ítems. Se contempla que una puntuación igual o superior a 6 indica la presencia de riesgo suicida (Rubio et al. 1998).

En la versión original reportó coeficientes de consistencia interna por ítem en un rango de $.84-.90$ y su fiabilidad test-retest es de .89. La adaptación a población española reportó una consistencia interna de .90 en la revisión de Sábado y Monforte (2010) fue de .81. 
En la revisión española (Sábado \& Monforte, 2010) reportaron la extracción de 4 factores con una varianza explicada del $52.64 \%$, se extrajeron mediante componentes principales y rotación varimax, el factor I denominado: sentimiento de inutilidad y quedo compuesto por los reactivos $3,6,8$ y 12 , el factor II denominado ideación suicida compuesto por los reactivos 2,13 y 14 , factor III denominado desesperanza, compuesto por los ítems 5,7 y 9, el factor IV denominado factores sociales: quedo compuesto por los reactivos $1,4,10,1115$. Se resalta que los autores no reportaron los índices de ajuste del factorial confirmatorio, por lo que para esta investigación se puso a prueba el factorial confirmatorio con el mismo modelo de la versión española.

\section{PROCEDIMIENTO}

Se contó con el aval de la Fiscalía de Reinserción Social y por el comité de bioética del Centro Universitario del Sur. Después de seleccionar los cinco centros penitenciarios, en cada institución se formaron grupos de entre 20 y 30 jóvenes en salones de usos múltiples. Antes de la aplicación de los instrumentos se solicitó que firmaran el consentimiento informado, para posteriormente proceder a la aplicación de los cuestionarios, mismos que fueron contestados en papel y lápiz. La aplicación de los instrumentos se realizó por el autor principal de éste trabajo.
Para evaluar la fiabilidad, consistencia interna y factorial de los instrumentos se calculó: Consistencia interna a partir del coeficiente Alpha de Cronbach. Para la bondad de ajuste en el factorial confirmatorio se tomaron como base, la raíz cuadrada media de error de aproximación (RMSEA < .06), la razón de chi-cuadrado sobre los grados de libertad $(\mathrm{CMIN} / \mathrm{DF}<3)$ y el índice global de bondad de ajuste (GFI > .9). Estos puntos de corte se tomaron de las propuestas de Schumacker y Lomax (2010), Byrne (2010), Kline (2011), Harrington (2009) y Manzano y Zamora (2009).

Los análisis estadísticos se realizaron con los programas: IBM Statistical Package for the Social Sciences Versión 24 (SPSS.24, 2016) y IBM Analysis of Moment Structures AMOS 18.

\section{RESULTADOS}

Del total de la muestra que contestó los instrumentos (Tabla 1), el $52.7 \%$ de los hombres $47.6 \%$ de las mujeres reportaron ser solteros respectivamente. Las mujeres mayor escolaridad $(41.3 \%$ educación media superior) y en los hombres fue la educación secundaria $(49.1 \%)$.

Tabla 1.

Variables sociodemográficas por sexo

\begin{tabular}{|c|c|c|c|c|c|c|}
\hline & & & & & & $\mathbf{p}$ \\
\hline & & $\mathbf{n}$ & $\%$ & $\mathbf{n}$ & $\%$ & \\
\hline Estado civil & & & & & & \\
\hline & Soltero & 175 & 52.7 & 30 & 47.6 & .161 \\
\hline & Unión Libre & 106 & 31.9 & 23 & 36.5 & \\
\hline & Casado & 12 & 3.6 & 2 & 3.2 & \\
\hline & Separado & 38 & 11.4 & 6 & 9.5 & \\
\hline & Viudo & 1 & 0.3 & 2 & 3.2 & \\
\hline Escolaridad & & & & & & \\
\hline & Educación Primaria & 96 & 28.9 & 8 & 12.7 & $.001 * * *$ \\
\hline & Educación Secundaria & 163 & 49.1 & 23 & 36.5 & \\
\hline & Educación Media Superior & 68 & 20.5 & 26 & 41.3 & \\
\hline & Otros & 5 & 1.5 & 6 & 9.5 & \\
\hline Edad por grupos & & & & & & \\
\hline & 15 a 19 años & 75 & 22.6 & 11 & 17.5 & .068 \\
\hline & 20 a 24 años & 89 & 26.8 & 26 & 41.3 & \\
\hline & 25 a 29 años & 168 & 50.6 & 26 & 41.3 & \\
\hline
\end{tabular}

Nota: El Valor de $\mathrm{p}$ se calculó mediante la prueba $\chi^{2}$ $\mathrm{p}<.05 * \mathrm{p}<.01 * * \mathrm{p}<.001 * * *$ 
Los coeficientes de fiabilidad calculados mediante el alfa de Cronbach (Tabla 2) se reportan tanto para la escala de riesgo de suicidio en general como para las submuestras de hombres y mujeres e incluso fueron calculados para cada uno de los factores.

Tabla 2.

Alfa de Cronbach de la Escala de Riesgo de suicidio por sexo

\begin{tabular}{lrrrrrr}
\hline & n & total & FI & FII & FIII & FIV \\
\hline Total & 395 & .749 & .562 & .555 & .259 & .441 \\
Hombres & 332 & .733 & .575 & .549 & .207 & .417 \\
Mujeres & 63 & .802 & .500 & .578 & .463 & .436 \\
\hline
\end{tabular}

Nota: FI=Factor I: Sentimiento de inutilidad. FII=Factor II: ideación suicida. FIII=Factor III: desesperanza. FIV=Factor IV: factores sociales.

La medida de adecuación muestral de KaiserMeyer-Olkin (.781) y la prueba de esfericidad de Bartlett con valor chi-cuadrado $=1093.80(\mathrm{gl}=105$, $\mathrm{p}<.001$ ) indican que los datos son adecuados para realizar un análisis factorial. La estructura propuesta (Figura 1) cuenta con cuatro factores, el porcentaje de varianza total explicado fue del $49.07 \%$, los índices de ajuste fueron; Ji2 $=223.914$, la raíz cuadrada media de error de aproximación $($ RMSEA < .06) = .065, la razón de chi-cuadrado sobre los grados de libertad (CMIN/ $\mathrm{DF}<3$ ) $=2.66$ y el índice de bondad de ajuste (GFI $>$.9) $=.927$. Las variancias, covariancias y cargas factoriales se presentan en la figura 1.

De acuerdo con los valores del factorial confirmatorio descritos en el párrafo anterior, muestra que la Escala de Riesgo de Suicidio tiene validez factorial con una varianza explicada del 49.7\%, así en la figura 1, se aprecia que los cuatro factores tienen una correlación estadísticamente significativa y positiva, las correlacionas más altas estuvieron entre sentimientos de inutilidad (factor I), ideación suicida (factor II) y factores sociales (factor IV).
Figura 1. Factorial confirmatorio de la Escala de Riesgo de Suicidio

Variancias

Covariancias

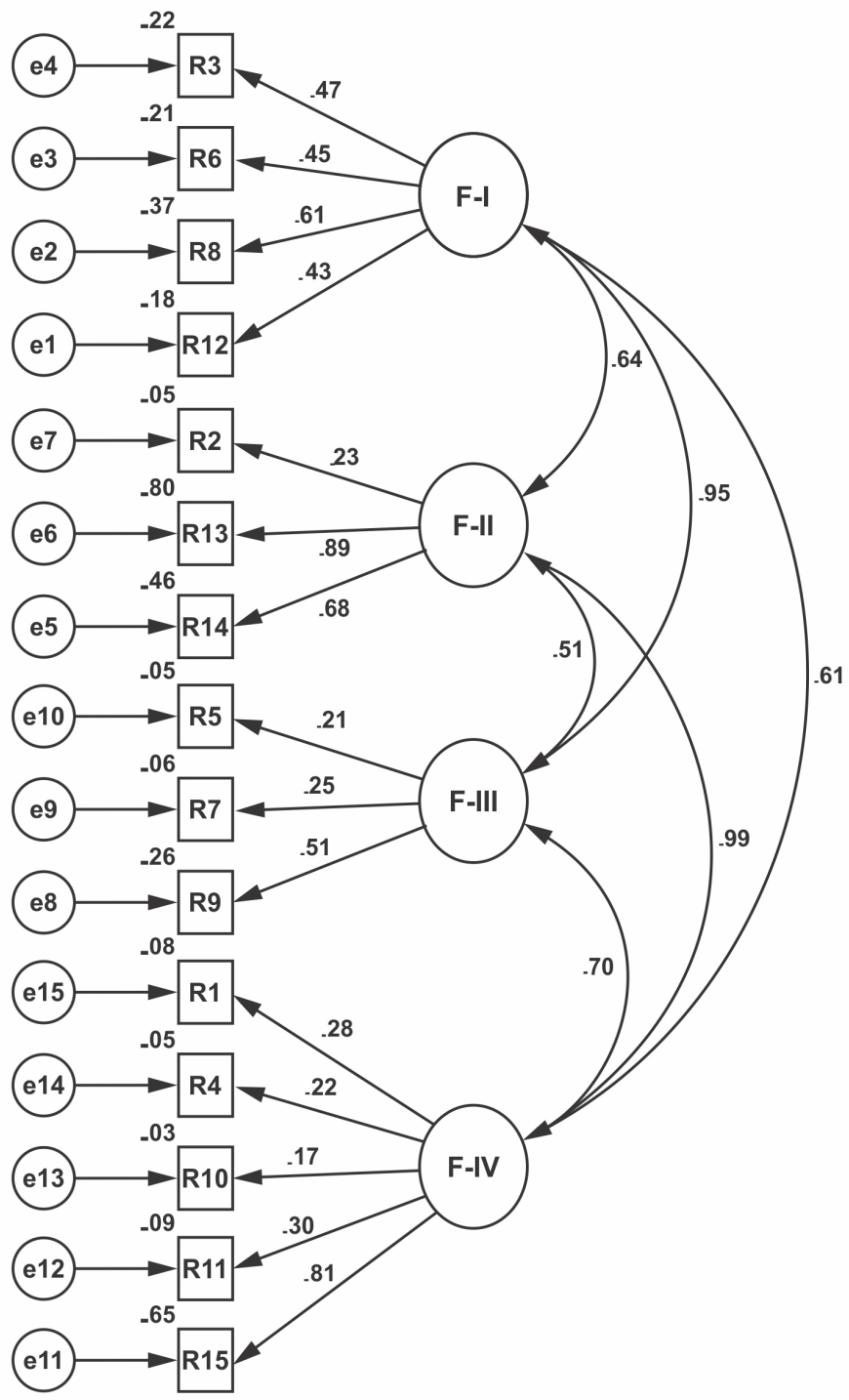

En la literatura se ha utilizado como punto de corte igual o superior a seis para determinar la presencia de riesgo de suicidio, en la tabla 3 se proponen los percentiles para determinar sin riesgo y riesgo alto, estos se calcularon tanto para escala en general como para cada uno de los factores. 
Tabla 3.

Percentiles para la escala de riesgo de suicidio $(n=395)$

\begin{tabular}{|c|c|c|c|c|c|c|}
\hline & Percentiles & $\begin{array}{c}\text { Escala de } \\
\text { riesgo de } \\
\text { suicidio }\end{array}$ & $\begin{array}{l}\text { Sentimientos } \\
\text { de inutilidad }\end{array}$ & $\begin{array}{c}\text { Ideación } \\
\text { suicida }\end{array}$ & $\begin{array}{c}\text { Deses } \\
\text { peranza }\end{array}$ & $\begin{array}{l}\text { Factore } \\
\text { sociale }\end{array}$ \\
\hline \multirow{5}{*}{$\begin{array}{l}\text { Sin } \\
\text { riesgo }\end{array}$} & 5 & 0 & 0 & 0 & 0 & 0 \\
\hline & 10 & 0 & 0 & 0 & 0 & 0 \\
\hline & 15 & 0 & 0 & 0 & 0 & 0 \\
\hline & 20 & 1 & 0 & 0 & 0 & 0 \\
\hline & 25 & 1 & 0 & 0 & 0 & 0 \\
\hline \multirow{5}{*}{$\begin{array}{l}\text { Riesgo } \\
\text { bajo }\end{array}$} & 30 & 1 & 0 & 0 & 0 & 0 \\
\hline & 35 & 2 & 0 & 0 & 0 & 0 \\
\hline & 40 & 2 & 1 & 0 & 0 & 0 \\
\hline & 45 & 2 & 1 & 0 & 0 & 1 \\
\hline & 50 & 2 & 1 & 0 & 0 & 1 \\
\hline \multirow{5}{*}{$\begin{array}{l}\text { Riesgo } \\
\text { medio }\end{array}$} & 55 & 3 & 1 & 0 & 0 & 1 \\
\hline & 60 & 3 & 1 & 0 & 0 & 1 \\
\hline & 65 & 4 & 2 & 1 & 1 & 1 \\
\hline & 70 & 4 & 2 & 1 & 1 & 1 \\
\hline & 75 & 5 & 2 & 1 & 1 & 1 \\
\hline \multirow{5}{*}{$\begin{array}{l}\text { Riesgo } \\
\text { alto }\end{array}$} & 80 & 6 & 2 & 1 & 1 & 2 \\
\hline & 85 & 7 & 3 & 2 & 1 & 2 \\
\hline & 90 & 8 & 3 & 2 & 1 & 2 \\
\hline & 95 & 9 & 4 & 2.2 & 2 & 3 \\
\hline & 99 & 13 & 4 & 3 & 3 & 5 \\
\hline \multicolumn{2}{|l|}{ Media } & 3.21 & 1.21 & 0.58 & 0.48 & 0.92 \\
\hline \multicolumn{2}{|l|}{$\begin{array}{l}\text { Desviación } \\
\text { estándar }\end{array}$} & 2.853 & 1.203 & .861 & 0.688 & 1.052 \\
\hline
\end{tabular}

\section{DISCUSIÓN}

Los índices de fiabilidad y confiabilidad reportados en esta investigación son consistentes con los reportados tanto en la versión original como en las adaptaciones españolas (Plutchik, Van Praag, Conte \& Ricard, 1989; Rubio et al. 1998; Sábado \& Monforte 2010). Ninguna de las investigaciones previas ha reportado diferencias por sexo, en esta investigación se reportan algunos resultados para cada una de las submuestras, en el caso de la propuesta de percentiles no se ofrecen separados, puesto que no existieron diferencias estadísticamente significativas.

Sábado y Monforte (2010) propusieron cuatro dimensiones dentro de la escala de riesgo de suicidio, con una varianza total explicada del $52.64 \%$, en el presente estudio, con los mismos factores, la varianza total explicada fue del $48.87 \%$, resultando ser aceptable y por primera vez se ofrece una propuesta de percentiles y baremos para población penitenciara.
La escala propuesta resulta útil para identificar los casos potenciales de riesgo de suicidio, puesto que engloba intentos suicidas previos, intensidad de ideación suicida actual, sentimientos de depresión y desesperanza y otros factores relacionados, mismos que pueden aumentar el riesgo de intentos futuros (Plutchik, Van Praag, Conte \& Ricard, 1989; RangelGarzón, Suárez-Beltrán y Escobar-Córdoba, 2015).

Los cuatro factores de la escala de riesgo de suicidio tuvieron cargas factoriales satisfactorias, y se correlacionaron entre sí, por lo que se determina que los sentimientos de inutilidad, ideación suicida, desesperanza y factores sociales, son factores que están relacionados con el riesgo de suicidio y una alta puntuación en estos incrementa la posibilidad de que ocurra la conducta suicida, esto es consistente con lo reportado por Beck, Steer, Kovacs y Garrison (1985) reportaron que la desesperanza es un predictor de los intentos de suicidio y esto es consistente con investigaciones recientes que identifican la desesperanza con un factor de riesgo para el suicidio (Serafini et al. 2017; Klonsky et al. 2012). Nuestros resultados son coherentes con otras investigaciones, puesto que se encontró una correlación significativa y positiva entre la escala de desesperanza y la escala de riesgo de suicidio.

Por último, estos resultados confirman lo reportado por Rangel-Garzón, Suárez-Beltrán y Escobar-Córdoba (2015), al referir que la Escala de Riesgo Suicida de Plutchik surgen como instrumento oportuno y confiable para la evaluación del riesgo suicida.

\section{CONCLUSIONES}

La escala de riesgo de suicido, demostró adecuadas propiedades psicométricas en población penitenciaria, por lo que resulta ser un instrumento idóneo para identificar los casos potenciales en situación de riesgo en esta población.

Por primera ocasión se ofrece una propuesta de baremos y percentiles para identificar a los sujetos con alto riesgo y sin riesgo de suicidio. 


\section{REFERENCIAS}

American Psychiatric Association (2014). Guía de consulta de los criterios diagnósticos del DSM-5. Editorial Medica Panamericana. Arlington, VA.

Aristizábal, C., Cañón, C., Castaño, J., Castaño, L., García, J., Marín, L., Osorno, C. \& SánchezValencia L. (2013). Riesgo suicida y factores asociados en instituciones de rehabilitación para adictos a las drogas en la ciudad de Manizales (Colombia). Archivos de Medicina (Manizales). 13(1) pp. 11-23. Disponible en: http://www. redalyc.org/articulo.oa?id=273828094002

Beck,A., Weissman, A., Lester, D. \& Trexler, L. (1974). The measurement of pessimism: the hopelessness scale. Journal Consulting and Clinical Psychology 42 (6), 861-865

Byrne, B.M. (2010). Structural Equation Modeling with AMOS. Basic Concepts, Applications, and Programming (2nd Ed). Routledge Taylor \& Francis Group New York, London.

Calvo-García, F., Giralt-Vázquez, C., Calvet-Roura, A. \& Carbonells-Sánchez, X. (2015). Riesgo de suicidio en población sin hogar. Clínica y Salud 27 (2016) 89-96. http://dx.doi.org/10.1016/ j.clysa.2016.05.002

Cañón, S. et al. (2015). Factor de riesgo suicida $y$ factores asociados en reclusas de un centro penitenciario de Caldas (Colombia), 2015: estudio de corte transversal. Manizales, Noviembre 2015.

Córdova, M. \& Rosales, J. (2010). La desesperanza de riesgo en jóvenes mexicanos y aspectos sociodemográficos asociados: diferencias por sexo. Psicología y Salud, 20(2) 195-201, juliodiciembre de 2010

Duthé, G., Hazard, A. \& Kensey, A. (2014). Trends and Risk Factors for Prisoner Suicide in France. Population-E, 69(4). doi: 10.3917/ pope.1404.0xxx

Fazel, S., Hayes, A.J., Bartellas, K., Clerici, M. \& Trestman, R. (2016). The mental health of prisoners: a review of prevalence, adverse outcomes and interventions. Lancet Psychiatry. September; 3(9): 871-881. doi:10.1016/S22150366(16)30142-0.
Fazel. S., Ramesh, T. \& Hawton, K. (2017). Suicide in prisons: an international study of prevalence and contributory factors. The Lancet Psychiatry. 4: 946-52. doi: 10.1016/S2215-0366(17)30430-3

Glenn,C.\& Nock, M. (2013). Improving the Prediction of Suicidal Behavior in Youth. International Journal of Law and Psychiatry; 36(0): 390-398. doi:10.1016/j.ijlp.2013.06.010

Harrington, D. (2009). Confirmatory Factor Analysis. Oxford University Press. New York.

Jaramillo, M., Silva, C., Rojas, B., Arango \& MedinaPérez, A. (2015). Ideación suicida y factores asociados en internos de un establecimiento penitenciario de Antioquia (Colombia). Revista colombiana de psiquiatría. 44(2):100-105 doi. org/10.1016/j.rcp.2015.01.006

Jiménez, M.P. \& Linero, L.M., (2015). Riesgo suicida, desesperanza y factores asociados en personal privado de la libertad de un Centro de Reclusión Militar en Colombia. R.F.S Revista Facultad de Salud.7(1): 68-75.doi: http://dx.doi.org/10.25054/ rfs.v7i1.182

Kline, R.B. (2011). Principles and Practice of Structural Equation Modeling (3rd Ed.). The Guilford Press. New York and London.

Manzano, A. \& Zamora, S. (2009). Sistema de ecuaciones estructurales: una herramienta de investigación. Cuaderno técnico 4. Centro Nacional de Evaluación para la Educación Superior, A.C. (Ceneval). México.

Marzano, L., Hawton, K., Rivlin, A. \& Fazel, S. $\square(2011)$. Psychosocial Characteristics and Social Networks of Suicidal Prisoners: Towards a Model of Suicidal Behaviour in Detention. PLoS ONE 8(7): e68944. doi:10.1371/journal. pone.0068944

Marzano, L., Hawton, K., Rivlin, A., Smith, N., Piper, M. \& Seena Fazel, S. (2016). Prevention of Suicidal Behavior in Prisons. An Overview of Initiatives Based on a Systematic Review of Research on Near-Lethal Suicide Attempts. Crisis 2016 doi: 10.1027/0227-5910/a000394

Mojica,C.,Sáenz,D.\&Rey-Anacona,C.(2009).Riesgo suicida, desesperanza y depresión en internos 
de un establecimiento carcelario colombiano. Revista Colombiana Psiquiatría. 38(4) 681-692. Disponible en: Disponible en: http://www.redalyc. org/articulo.oa?id=80615450009

Mondragón, L. (1997). La ideación suicida en relación con la desesperanza, el abuso de drogas y alcohol. Tesis de licenciatura en psicología. Universidad Nacional Autónoma de México, México, D.F.

National Action Alliance for Suicide Prevention: Youth in Contact with the Juvenile Justice System Task Force. (2013). Suicidal ideation and behavior among youth in the juvenile justice system: A review of the literature. Washington, DC: Author.

Plutchick, R., Van Praag, H, Conte, H. \& Picard, S. (1989). Correlates of Suicide and Violence Risk I: The Suicide Risk Measure. Comprehensive Psychiatry 30(4): 296-302.

Plutchick, R.\& Van Praag,H.(1989). The measurement of suicidality, aggressivity and impulsivity. Prog. Neuro-Psychophormaco. 6(13) 23-34 Printed in Great Britain. All rights reserved.

Radeloff, D., Lempp, T., Herrmann, E., Kettner, M., Bennefeld-Kersten, K. \& Freitag, C.M. (2015).
National total Survey of German adolescent Suicide in Prison. European Child \& Adolescent Psychiatry 24:219-225 doi 10.1007/s00787-0140568-1

Rangel-Garzón, CX., Suárez-Beltrán, MF. \& EscobarCórdoba F. (2015). Escalas de evaluación de riesgo suicida en atención primaria. Revista Facultad de Medicina 63(4):707-16. doi: http://dx.doi. org/10.15446/revfacmed.v63.n4.50849.

Rubio G, Montero I, Jáuregui J, Villanueva R., Casado MA, Marín J.J. \& Santo-Domingo J. (1998). Validación de la escala de riesgo suicida de Plutchik en población española. Archivos de Neurobiología 61(2): 143-52.

Sábado, J.T. \& Monforte, C. (2010). Propiedades métricas de la escala de riesgo suicida de Plutchik en profesionales de enfermería. $11^{\circ}$ Congreso Virtual de Psiquiatría. Interpsiquis 2010.

Schumacker, R.E. \& Lomax, R.G. (2010). A Beginner's Guide to Structural Equation Modeling (3rd Ed.). Routledge Taylor \& Francis Group New York, London.

Fecha de recepción: 1 de junio, 2018

Fecha de aceptación: 4 de julio, 2018 